\title{
Teoría Queer: ¿Cambio de paradigma, nuevas metodologías para la investigación social o promoción de niveles de vida más dignos?
}

Queer Theory: Change of paradigm, new methodologies for social research or an encouragement for better life standards?

Teoria Queer: mudança de paradigma, novas metodologias para investigação social ou a promoção de níveis de vida mais dignos?

\section{Ingrid Ambrosy}

Universidad Rafael Landívar. Telf.: (502) 2477-2163. Correo electrónico: iambrosy@url.edu.gt

\section{RESUMEN}

Este artículo trata sobre la teoría queer, desarrollada a partir de los años 80, y sus implicaciones en la manera de pensar y de investigar sobre el tema de identidad de sexo y género, la cual conlleva una nueva visión del humanismo, donde pueden ser aceptadas las diferencias individuales y grupales, así como una metodología de investigación que pueda ser útil para los estudios en este campo.

Palabras clave: humanismo crítico, teoría queer, investigación cualitativa.

\begin{abstract}
This article deals with queer theory, which has been developed since the 1980s, and its implications in the way of thinking and research on the topic of identity of sex and gender. Entailing a new vision of humanism, where individual and group differences can be accepted as well as a research methodology that may be useful for the studies in this field.

Key words: critical humanism, queer theory, qualitative research.

\section{RESUMO}

Trata-se da teoria queer, desenvolvida a partir dos anos 80, e de suas implicações na maneira de pensar e de pesquisar sobre o tema identidade sexual e gênero, o que envolve uma nova visão do humanismo e de onde podem ser aceitas as diferenças individuais e grupais, bem como uma metodologia de investigação que pode ser útil para os estudos neste campo.
\end{abstract}

Palavras chave: humanismo crítico, teoria queer, pesquisa qualitativa. 


\section{INTRODUCCIÓN}

En los últimos 50 años se han visto más cambios que en cientos de años atrás. En todas las esferas de la vida, como lo es en la economía, la política, las ciencias y la educación, entre otros, constantemente surgen nuevas perspectivas, que influyen directamente la forma de pensar, pero también de actuar de las personas. Vivimos en la era de la globalización, donde los lazos económicos y políticos no tienen fronteras, al igual que las tecnologías de la información y comunicación, con lo que el conocimiento, las relaciones interpersonales, los movimientos financieros y políticos se pueden realizar en un tiempo y en un espacio que no tiene límites, nada más los que imponen las condiciones de acceso al sistema. Como consecuencia, la nueva sociedad global también debe permitir que todas las personas sean incluidas para participar democrática y activamente ante las demandas del mundo (Resnik, 2007).

Otra de las características de la época actual es que hay mucha más movilidad social y profesional, consecuencia también de la globalización, sin embargo, esto ha promovido la tendencia entre grupos sociales a compararse unos con otros; aun aquellos que cuentan con diversos satisfactores y que se encuentran en las condiciones más aceptables para la sociedad, lo hacen, constituyéndose dicha comparación en fuente de infelicidad e inseguridad (Hobsbawm, 2007).

En el plano social, también ha habido cambios debido al interés por la igualdad y equidad entre grupos de distintas denominaciones o pertenecientes a minorías. Asimismo, hay más participación ciudadana para demostrar sus propias posturas, para valorar sus experiencias y para demandar el cumplimiento de sus derechos. Es así como, uno de los temas más controversiales y más estudiados en los últimos años (Toro-Alfonso, 2005), como lo es la sexualidad, es abordado con más libertad en estos tiempos y surgen múltiples puntos de vista sobre las clasificaciones que se han hecho de la misma, y sobre lo que es aceptado o no en las diferentes sociedades. Esto despierta nuevos intereses para la investigación, en especial para investigadores que se han visto afectados en su experiencia personal por lo que la sociedad y otras investigaciones afirman o confirman sobre el tema.

El tema de la sexualidad es abordado no sólo socialmente, sino también en muchas investigaciones como una condición donde el sexo físico y la orientación sexual son los únicos componentes de ella, y si las personas salen de la normalidad (homosexualidad, hermafroditismo, expresión corporal no identificada socialmente con su sexo), o lo que es socialmente aceptado por la mayoría (heterosexualidad, hombre o mujer, expresión corporal socialmente acorde al sexo), se estudian sus causas, sus comportamientos y son vistos como personas "raras".

Sin embargo, también se ha creado, aunque todavía falta mucho, una mayor conciencia sobre las diferencias y el respeto a las mismas, porque es una condición necesaria para que las personas alrededor del mundo tengan una mejor convivencia, ya que las consecuencias de la poca tolerancia a la diversidad han generado circunstancias de inequidad, desigualdad, violencia y muerte, que van en contra de la paz tan anhelada a través de los tiempos.

Dentro de los grupos que se han revelado, en contra de un abordaje de la sexualidad que encierra a las personas en una de las combinaciones aceptadas por la sociedad, se encuentran los seguidores de la teoría queer, la cual da un giro radical a las categorías convencionales de sexualidad y género. 
En las siguientes líneas se abordará la teoría queer para vislumbrar si consiste en un cambio de paradigma, si más bien se refiere a nuevas metodologías para la investigación social o si su principal función es la promoción de niveles de vida más dignos en la sociedad global.

\section{LA COMPLEJIDAD SEXUAL DEL SER HUMANO}

La sexualidad es un aspecto intrínseco al ser humano. Desde que nace, durante todo su proceso de formación (dentro de la familia y en las instituciones educativas), así como en todos los contextos en los que se desenvuelve, la persona manifiesta y aprende sobre la sexualidad (Papalia, Old \& Wendknos, 2006). Sin embargo, la influencia de la cultura asigna a cada sexo un género y a cada género un rol diferente. En consecuencia, según se sea hombre o mujer, la persona adopta las características que, de acuerdo con su género, son aceptadas por la sociedad en la que se desarrolla.

De acuerdo con Mayobre (2006), "tradicionalmente se consideraba que el sexo era el factor determinante de las diferencias observadas entre varones y mujeres y que era el causante de las diferencias sociales existentes entre las personas sexuadas en masculino o femenino". Sin embargo, como menciona la autora, para la conformación de la identidad, sea masculina o femenina, están presentes, además, factores genéticos, estrategias de poder, elementos simbólicos, psicológicos, sociales, culturales, entre otros, por lo que la genética no es la única que contribuye en la configuración de la identidad personal. Por lo tanto, las personas construyen su masculinidad o feminidad luego de un largo proceso, donde el medio familiar y social tiene sus implicaciones.

Las diferencias en cuanto a roles transcienden la vida personal, puesto que luego, en el seno de la familia y en todos los demás ambientes sociales, las diferencias han trazado una distancia entre lo que debe ser y hacer cada género y en cómo debe ser tratado. Como resultado, históricamente se ha visto que la balanza se inclina a favor de las personas que muestran coherencia entre su sexo-género-orientación sexual y se genera un rechazo hacia las personas que tienen una identidad sexual diferente a su sexo, que su orientación sexual es diferente a la esperada, dado su sexo, o quienes conductualmente se manifiestan de forma diferente.

La cantidad de personas que salen de los parámetros impuestos por la sociedad cada vez es mayor o, al menos, se conoce más de ellas y hay más libertad para expresarse y exigir sus derechos. Por lo mismo, la necesidad de investigar en este campo se hace más grande, hay más preguntas que respuestas, y la investigación debe incurrir de la forma más apropiada, para el bien de la humanidad. De acuerdo con Toro-Alfonso (2005), en esta época hay carencia de estudios que ayuden a comprender el significado y la complejidad que los seres humanos le adjudican a la sexualidad. Los hay sobre frecuencias y relaciones con factores de riesgo, pero no sobre la diversidad que conforma la sociedad contemporánea y es necesario trabajar en esta línea para comprender más a la sociedad actual y para contribuir a la disminución de luchas sociales y al cumplimiento de los derechos de todas las personas. 


\section{HUMANISMO Y EL ABORDAJE DEL TEMA SEXO-GÉNERO}

El humanismo es un vocablo donde muchos autores tienen opiniones divergentes, puesto que se desarrolló en un período muy largo que viene desde la época renacentista hasta la modernidad. Sin embargo, aunque para los distintos autores tenga significados diferentes, ya sea por su disciplina o momento histórico en que surgió, en la base del humanismo se encuentra una tendencia a colocar al ser humano como el valor principal del mundo, así como a afirmar la igualdad de los seres humanos, reconocer la diversidad personal y cultural, desarrollar el conocimiento por encima de las verdades consideradas como absolutas, afirmar la libertad de ideas y creencias, repudiar la violencia, y tener una relación consciente con la naturaleza (Aguirre, 2002); es decir, hacer al hombre más humano, utilizando para ello sus propias potencialidades personales y las ciencias, como herramientas para construir su libertad (Maritain, 2001).

Aunque el humanismo ha tenido un fuerte impacto en la promoción de valores para una sociedad más humana, sus estudios ubicaban a las personas dentro de grupos con características que podían ser generalizables a grupos más amplios y, en muchos de los casos, los resultados de las investigaciones no podían ser aplicables a otras personas. Las formas para recabar información fueron las historias de vida, la observación participante y los grupos focales (Plummer, en Denzin y Lincoln, 2005). Especialmente, personas que salen de "los parámetros socialmente aceptables" quedan fuera o son considerados como personas diferentes, por lo que el abordaje del tema de género y sexo debe ser manejado de forma diferente y ello implica una nueva forma de pensar sobre las clasificaciones que se han hecho en el tema de la sexualidad.

Si durante el humanismo se buscaba generalizar datos y se analizaba la diversidad sexual como algo fuera de la norma, en el humanismo crítico se busca que las diferencias sean estudiadas, pero no como algo extraño o enfermizo, sino como una forma más de ser que merece respeto en su forma de ser, pensar y actuar, así como un trato humano y en igualdad de derechos y obligaciones.

Dooyeweerd (1998) enfatizó esta realidad, resaltando que las ciencias deberían contribuir con la libertad y justicia de las personas, ya que si ese objetivo no se lograba, se ponía en duda el valor de las mismas. Este es un punto a favor de una nueva mirada a la diversidad sexual, que abrirá el camino para realizar estudios con un enfoque humanista diferente, cuyas conclusiones ayudarán a la sociedad a construir un ambiente de paz en la diversidad. Las ciencias humanas no pueden ser ni ciencias ni humanas si no consideran al ser humano real (no al ideal) y si no trabajan en conjunto diversas disciplinas (Rubio, 1987). Esta es una lección que deja el humanismo crítico, con lo que se espera los estudios basados en la teoría queer colaboren en la construcción de un mundo más humano.

\section{TEORÍA QUEER, CAMBIO EN LA CONCEPTUALIZACIÓN DE LA SEXUALIDAD}

El término inglés queer "designa la idea de rareza y extrañamiento", aunque también, de una manera coloquial, se ha utilizado como un "insulto sexual dirigido tanto contra hombres como contra mujeres" que demuestran comportamientos sexuales que salen de los parámetros socialmente aceptados (Mérida, 2002). 
La teoría queer implica un cambio en la forma en que hasta el momento se ha pensado sobre el sexo y género. De acuerdo con Córdova (2003), a través de esta teoría se pretende reactivar y reforzar que la identidad sexual es un tema que debe llevarse al plano social, donde se debe renunciar a la reivindicación de la normalidad, puesto que el binomio normal-patológico, en la identidad sexual, debe perder fuerza. ¿Y por qué debe perder fuerza? Porque las diferentes expresiones de la sexualidad ya no se ajustan a una descripción estrecha donde debe encasillarse a las personas en alguno de los polos normalidad-patología. La idea de una única sexualidad ideal es característica de la mayoría de los sistemas de pensamiento sobre el sexo (Rubin, s.f.), sin embargo, la teoría queer invita a ver el mundo sin categorías (Careaga ,2003).

En la conformación de cada persona coexisten su sexo biológico, su identidad de género, su expresión de género y su orientación sexual; todas estas dimensiones que mantienen relaciones de distinto orden y sincronía dentro de un cuerpo que cuando problematizan la norma heterosexual desafían las concepción de género que ha sido aceptada hasta épocas recientes para la construcción de la feminidad o la masculinidad (Espinosa, 2008). La teoría queer implica pues un cambio en la manera de pensar sobre la sexualidad y, por lo mismo, en la búsqueda de información que pueda ayudar a comprender este tema. Podría decirse también que consiste en "un desciframiento postmodernista de la noción de identidad sexual que enlaza históricamente con el camino abierto por el feminismo, los estudios sobre la mujer o sobre el género", pero que promueve nuevas metodologías para su estudio, incluyendo la deconstrucción del binomio "hetero/homo" (Mérida, 2002).

\section{METODOLOGÍAS PARA ESTUDIOS QUEER}

Para hacer investigación queer, uno de los primeros pasos a realizar es la revisión de la propia postura del investigador ante el tema de estudio. Al respecto, Careaga (2003) señaló que al aproximarse al estudio de la diversidad sexual el investigador deben revisar sus propios conceptos sobre sexualidad, y evaluar si considera el término más allá de la genitalidad, y si puede valorar las tres dimensiones que dentro del contexto queer se utilizan para su análisis y definición: la orientación sexual, la identidad sexual y la expresión sexual.

Los estudios queer tienen como finalidad romper con los esquemas de desigualdad, discriminación y opresión, entre otros, que caracterizan a las minorías en las sociedades de hoy, pero especialmente a las relacionadas con la sexualidad. No se lucha por un derecho a la intimidad, sino por la libertad pública de ser quien se es (Mérida, 2002). Asimismo, dichos estudios revisan las tensiones entre la afirmación de las identidades políticas (lesbianas, gays, bisexuales, transexuales) y la deconstrucción de las mismas (Gómez, 2007).

En dicha línea, Seidman (1996) considera que el estudio de la teoría queer está más encaminado a la consideración de cuáles deberían ser la políticas que deben adoptarse ante la diversidad sexual, especialmente para contribuir en distintos ámbitos sociales, entre ellos las interacciones sociales y la salud.

De acuerdo con Gómez (2007), existe un desafío al pensar en los métodos y en los supuestos teóricos sobre el estudio de la sexualidad, y es que cuando se aborda el tema 
se debe pensar siempre en los aportes que de ella se derivarán. Plummer (2005) es uno de los pocos autores que describe algunas de las metodologías de investigación utilizadas dentro de la teoría queer. En general, todas las técnicas pertenecen a la investigación cualitativa. Una de las técnicas que menciona es la relectura de artefactos culturales, la cual consiste en hacer un análisis de los textos, los cuales pueden tomarse de filmes, de la literatura, la televisión, una ópera o de musicales, siendo ésta una de las estrategias más utilizadas. También suele ser utilizada la etnografía revolucionaria, la cual se desarrolla a través de un trabajo de campo donde el investigador puede ser parte de la investigación, aportando su propia experiencia.

Otra de las estrategias es el montaje de un texto analítico basándose en la recopilación de diferentes textos, los cuales pueden surgir de diversas fuentes. Asimismo, también se puede utilizar el método del análisis de las actuaciones sobre género, especialmente las relacionadas con grupos de travestis y personas transgénero. Por otro lado, puede acudirse también a la exploración de nuevos casos de estudio asociados con la teoría queer y a la lectura del sí mismo del autor, que puede estar plasmado en musicales, textos y fotografías.

Como puede verse, la teoría queer se vale de diferentes estrategias para lograr comprender la profundizar de la categoría género desde los textos, los filmes, las personas y hasta en el mismo autor.

Para Thompson (2007), a través de la metodología queer se puede hacer una crítica al estilo que se observa en los artefactos culturales que demuestran tendencias sobre la conceptualización del género en la sociedad, lo cual puede ser usado en discusiones serias sobre cuestiones políticas y sociales. Sin embargo, no sólo pueden utilizarse con esos fines, sino que también pueden servir para generar consciencia en la sociedad sobre temas de interés actual para la mejor convivencia, como lo son la diversidad, el respeto a los derechos humanos, la libertad, la justicia y la igualdad, temas que en el sector educativo pueden ser tratados.

\section{DISCUSIÓN}

¿La teoría queer representa un cambio de paradigma o es más bien un llamado para hacer la investigación sobre el género y sexo desde otras metodologías? ¿Es un reto para la sociedad que busca el respeto a la libertad y la diversidad, así como la igualdad, la justicia y niveles de vida más dignos para todos los seres humanos?

De manera general, podría decirse que los estudios queer representan un reto para las ciencias, para la política, para la investigación e incluso para la educación. Primero porque rompe los esquemas sobre las clasificaciones que hasta ahora se habían hecho sobre el sexo y el género, donde no todas las personas caben, especialmente porque rompe con la hegemonía que aún mantiene la heterosexualidad dentro de la orientación sexual, así como la expresión conductual de los roles asignados a cada uno de los géneros, que hasta ahora son: masculino y femenino.

Por otro lado, implica un replanteamiento de las formas que hasta el momento se han utilizado para estudiar el tema de la sexualidad. Además, representa para la educación una forma de contribuir con la formación de los ciudadanos de esta era que se desenvuelven en un mundo global, donde la diversidad está presente en todas las relaciones 
que se establecen y que, si no se sabe manejar, puede desestabilizar el equilibrio y la paz entre los seres humanos.

La mayoría de autores que han analizado el tema (Mérida, 2002, Córdova, 2003; Careaga, 2003; Rubin, s.f.) coinciden en que el estudio de la sexualidad debe partir de un cambio sobre la forma como se conceptualiza la sexualidad. Este es un factor clave, pero aún no podría hablarse de un cambio de paradigma, puesto que no hay acuerdos entre grupos de científicos ni hay una metodología específica que sea reconocida para hacer investigación desde la teoría queer. Sin embargo, a futuro es probable que haya un cambio de paradigma, puesto que para hacer estudios sobre sexualidad se tendrá que tomar un fundamento teórico y mientras más consensos haya sobre las bases de la teoría queer, más cerca se estará de ese cambio de paradigma.

En segundo lugar, las metodologías que pueden ayudar a construir la investigación queer van más allá de las historias de vida, la etnografía y la observación participante, que en otros estudios tienden a ver a las personas como parte de un grupo donde pueden generalizarse sus resultados, como se hacía desde el humanismo. La teoría queer se vale de análisis de textos, que pueden encontrarse en películas, literatura, televisión, musicales u óperas; de una etnografía revolucionaria, de la elaboración de textos luego de la revisión de otros que pueden ser de distinto formato; de casos de estudio, representaciones de género (videos, teatro callejero) e incluso de la investigación de los autores de obras musicales, teatrales o literarias. Todas estas metodologías invitan a abordar de una forma más creativa e integradora el tema de género y sexo. Sin embargo, algunas de estas estrategias ya eran utilizadas en el campo de las humanidades, pero en su mayoría son utilizadas con nuevos aires, con una interpretación más apegada a los estilos y a las formas en que los datos obtenidos reflejan ideas asociadas a sexo y género; sin olvidar que los resultados siempre tendrán sentido para la sociedad en la medida que motiven a un actuar diferente.

Respecto de la importancia de utilizar una metodología acorde al tema queer, los autores presentan diferentes puntos de vista sobre el por qué hacer investigación queer y todas son válidas, por ejemplo Mérida (2002) enfatiza el derecho a expresarse como se es, Seidman (1996), el papel de los resultados en las políticas sociales, y Córdova (2003) propugna un cambio en la representación binomial del sexo-género; sin embargo, aunado a esos objetivos específicos, más allá de la utilización de ciertos resultados para determinados fines, el hacer una investigación adecuada, con la metodología más adaptable a los estudios queer, finalmente ayudarán a obtener resultados que puedan devolverle a la sociedad un reflejo de lo que en ella sucede para provocar cambios desde las personas hacia sus relaciones con los demás y desde las estructuras sociales, para que se fomente el respeto a la diversidad.

En educación hará falta un largo recorrido para conocer y profundizar en el tema, pero es un camino que debe hacerse, especialmente porque la sociedad globalizada requiere ciudadanos libres, solidarios, justos y honestos, y si dentro del sistema educativo se abordan estos temas, basados en las últimas investigaciones y motivando a hacer más investigación, los prejuicios, las discriminaciones y la violencia a determinados grupos pueden frenarse. La teoría queer debe ser un tema que forme parte de la agenda educativa porque los aportes de la misma pueden apoyar el desarrollo de una flexibilidad cognoscitiva y emocional para abordar el tema de la sexualidad y con ello incidir en un mundo de paz. 
Y en tercer lugar, al lograr una investigación más profunda del tema, se puede comprender más la complejidad del ser humano y consecuentemente respetar más las diferencias, la libertad de las personas. Esto es un pilar importante para lograr la justicia y el respeto por la dignidad. Así que, aunque la teoría queer de momento no implica un cambio de paradigma en la concepción que tenemos del sexo y del género, la misma investigación realizada con métodos variados que exploran de diferentes formas el simbolismo y las características de la sexualidad, todos los esfuerzos que se realicen por profundizar en este tema al final constituirán una herramienta más para la construcción de un mundo más humano.

Por lo tanto, la teoría queer ofrece la utilización de diversas metodologías cualitativas para logar sus objetivos, y aunque en este artículo no se profundiza en las mismas, puesto que dicho estudio sería parte de otro artículo, con ellas se brinda la oportunidad de lograr el respeto por la diversidad y esto constituye un gran aporte de la investigación al brindar información que puede servir para lograr muchos de los acuerdos mundiales vigentes en este momento relacionados con la justicia, equidad y respeto por la diversidad. Es muy probable que, con el tiempo, los métodos usados para la investigación queer constituyan un referente para otros campos sociales relacionados con minorías, aunque de momento hace falta sistematizar y profundizar más en los que utiliza para su investigación.

\section{REFERENCIAS BIBLIOGRÁFICAS}

Aguirre, L. (2002). Educación superior, compromiso social y humanismo crítico. Tercer Congreso Nacional y Segundo Internacional. Retos y Expectativas de la Educación Superior. Instituto de Investigación y Desarrollo Educativo.

Careaga, G. (2003). Aproximaciones para el estudio de la diversidad sexual. Sexología y sociedad, a.9, n.22.

Córdova, D. (2003). Identidad sexual y performatividad. Athenea Digital, n.4, 87-96.

Denzin, N. (2008). Los nuevos diálogos sobre paradigmas y la investigación cualitativa, un compromiso en la relación universidad-sociedad. Reencuentro, n.52, 63-76.

Dooyeweerd, H. 1998. La Raíces de la cultura Occidental. Barcelona: CLIE.

Espinosa, B. (2008). Cuerpos y diversidad sexual: aporte para la igualdad y el reconocimiento. Colombia: Pontificia Universidad Javeriana.

Gómez, M. (2007). Presentación. Revista de estudios sociales, n.28, 11-13.

Hobsbawm, E. (2007). Entrevista sobre el siglo XXI. España: Crítica.

Maritain, J. (2001). Humanismo integral. (2 ${ }^{\mathrm{a}}$. Ed.). España: ediciones palabra.

Mayobre, P. (2006). La formación de la Identidad de Género: Una mirada desde la filosofía. En Zarazaga, E. et al., Educación Social e Igualdad de Género. (pp. 21-59). Málaga: Ayuntamiento de Málaga.

Mérida, R. (2002). Sexualidades transgresoras: una antología de estudios queer. Barcelona: Icaria Editorial S.A. Universidad Autónoma de Baja California.

Papalia, Old \& Wendknos, (2006). Desarrollo Humano. México: McGraw-Hill Interamericana.

Plummer, K. (2005). Critical Humanism and queer theory. En Denzin, N. y Lincoln, Y. The Sage Handbook of Qualitative Research. London: Sage.

Resnik, P. (2007). La democracia del siglo XXI. España: Anthopos.

Rubin, G. (s/f). Reflexionando sobre el sexo: notas para una teoría radical de la sexualidad. Biblioteca virtual de Ciencias Sociales. Disponible en: http://webs.uvigo.es/xenero/profesorado/ beatriz_suarez/rubin.pdf. 
Rubio, J. (1987). El hombre y la ética. España: Anthropos.

Seidman, S. (1996). Queer Theory/Sociology. Cambridge: Blackwell Publisers.

Thompson, R. (2007). What's Queer about Race? South Atlantic Quarterly. Summer, pp. 478-483.

Toro-Alfonso, J. (2005). El estudio de las homosexualidades: revisión, retos éticos y metodológicos.

Revista de ciencias sociales, n.14, 78-94. 